\title{
Product Transform
}

Benedict Irwin ${ }^{1}$

${ }^{1}$ University of Cambridge

January 7, 2021

Consider a transform that acts on a function to extract the product series coefficient. For example if a function allows a series expansion

$$
f(x)=\prod_{k=1}^{\infty}\left(1+a_{k} x^{k}\right)=\sum_{k=0}^{\infty} b_{k} x^{k}
$$

then we define the transform $K$ of $f$ to be

$$
K_{x}[f](s)=a_{s}
$$

likewise the inverse transform gives

$$
K_{s}^{-1}\left[a_{s}\right](x)=f(x)
$$

then by definition of the Pochhammer-q function we have

$$
\begin{array}{r}
K_{x}\left[\frac{1}{2}(-1, x)_{\infty}\right]=1 \\
K_{x}\left[(q, q)_{\infty}\right]=-1
\end{array}
$$

then certain functions with number theoretic potential can be defined by the inverse transform

$$
\begin{aligned}
& K_{s}^{-1}[\lambda(s)] \\
& K_{s}^{-1}[\mu(s)] \\
& K_{s}^{-1}[\phi(s)] \\
& K_{s}^{-1}[\Omega(s)]
\end{aligned}
$$

for functions $\lambda(s), \mu(s), .$. the Liouville function, Moebius function, totient function etc. If we attempt to extract the coefficeints of a well known function, for example

$$
\exp (x)=(1+1 x)\left(1+\frac{x^{2}}{2}\right)\left(1-\frac{x^{3}}{3}\right)\left(1+\frac{3 x^{4}}{8}\right)\left(1-\frac{x^{5}}{5}\right)\left(1+\frac{13 x^{6}}{72}\right) \cdots
$$

we find that the coefficients of odd prime powers $p$ are $-1 / p$. We find that

$$
\mathcal{K}_{x}\left[\frac{1}{1-x}\right](s)=\chi_{2^{k}}(s)
$$

which is the characteristic function of non-zero powers of 2 , which comes from the interpretation of that number of ways to partition each number into powers of 2 is 1 . 
We have

$$
b_{7}=\left(a_{1} a_{2} a_{4}+a_{3} a_{4}+a_{2} a_{5}+a_{1} a_{6}+a_{7}\right)
$$

which is clearly a sum over the ways to make 7 from unique choices of $k$. Whereas terms like

$$
a_{5}=b_{5}-b_{2} b_{3}+b_{1} b_{2} b_{2}-b_{1} b_{4}+b_{1} b_{1} b_{3}-b_{1} b_{1} b_{1} b_{2}
$$

where the signs seem to correspond to the number of terms and repeats are now allowed. This gives

$$
b_{n}=\left(\sum_{k_{1}=1}^{n}\left[k_{1}=n\right] a_{k_{1}}\right)+\left(\sum_{k_{1}=1}^{n} \sum_{k_{2}>k_{1}}^{n}\left[k_{1}+k_{2}=n\right] a_{k_{1}} a_{k_{2}}\right)+\cdots
$$

and then

$$
a_{n}=\left(\sum_{k_{1}=1}^{n}\left[k_{1}=n\right] b_{k_{1}}\right)-\left(\sum_{k_{1}=1}^{n} \sum_{k_{2}=1}^{n}\left[k_{1}+k_{2}=n\right] g_{k_{1} k_{2}} b_{k_{1}} b_{k_{2}}\right)+\cdots
$$

where the $g_{k l}$ correct for multiplicities. 